\title{
The good, the bad and the really ugly: composite-spectrum binaries
}

\author{
R.E.M. Griffin \\ Herzberg Astrophysics 83 Astronomy Research Centre, NRC, \\ 5071 West Saanich Road, Victoria, BC, V9E 2E\%, Canada \\ (E-mail: elizabeth.griffin@nrc-cnrc.gc.ca)
}

Received: November 7, 2019; Accepted: January 24, 2020

\begin{abstract}
Composite-spectrum binaries (containing a cool giant primary and a hot dwarf secondary) should be an answer to a theoretician's prayer. Because of their luminosity difference, both spectra are visible in the near UV, a region that includes several valuable luminosity and temperature indicators. If we just measure the radial velocity (RV) of the secondary at different dates, and construct an SB2 orbit, we immediate get the mass ratio of the component stars. A guess of the mass of the dwarf, and one thence obtains the mass of the cool giant - a unique and immensely valuable method. But just measure a hot dwarf's RV?? It raises many problems, mostly caused by the nature of the star. A hot (late-B or early-A) dwarf has few lines, most are weak, they can be very blurred by rotation, and they get hidden by the crowded spectra of the giant. Spectral subtraction works a treat in separating the two spectra, but the residue is inevitably rather noisy. Nevertheless, results from well over half of the 45 brightest northern composite-spectrum binaries have been published; about 1 in 6 are triple systems, and a few have characteristics that defy any theoretician to explain. Several are 'bad' because their giant primaries have high luminosity and finding a matching standard for the subtraction process is troublesome, while the 'really ugly' include an Am star in a simple SB2 binary with a period of 75 years, a pair of early-A dwarfs in a 3-day orbit with amplitudes of $100 \mathrm{~km} \mathrm{~s}^{-1}$ and which show absolutely no rotational broadening at all, and another whose secondary (apparently a single star) is more that twice as massive as its primary giant. But 8 of the sample are also eclipsing, and manifest the hugely important phenomenon of chromospheric absorption - those are the 'really good' systems. The talk showed examples of each kind. Key words: stars: binaries - stars:composite-systems - stars:stellar evolution
\end{abstract}

\section{Introduction}

Composite-spectrum binaries, whose component stars are a cool (G-K-M) giant and a hot (B-A, and maybe F) dwarf, form an important sub-branch of spectroscopic binaries because they exhibit the spectra of both component stars (a property that was recognized over 100 years ago by the classifiers of the Henry 
Draper Catalogue). Measurements of both spectra enable SB2 orbits to be constructed, and thence the mass ratio of the component stars to be determined. Since stellar mass is the property that controls the rate at which a star evolves, the star that is now a cool giant must be the more massive of the two, and thereby the more luminous. But although the spectral energy distributions of the two stars are of course highly different, their gradients are such that, in the near-UV and blue spectral regions, they are tolerably comparable. Both spectra can therefore been seen in those wavelength regions, so the mass ratio can be determined. It then requires an estimation of the mass of the hot dwarf (the latitude of those values is quite small) in order to determine, with reasonable precision, the mass of the giant star - certainly a much-welcomed determination of a property that is otherwise particularly elusive.

An ongoing project to study composite-spectrum binaries is working with a sample of 45 of the brightest systems visible from the northern hemisphere (see Table 1). Analyses of well over half have now been published - for example, Griffin \& Griffin (1986) (Paper 1), or Griffin \& Griffin (2018) (Paper 22), among others, those remaining are necessarily 'difficult' in some respect.

Table 1. Table 1. Statistics of the sample of 45 binaries

\begin{tabular}{lcc}
\hline \hline & $\begin{array}{c}\text { Cool primary } \\
(\text { evolved })^{* *}\end{array}$ & $\begin{array}{c}\text { Hot secondary } \\
\text { (much less evolved) }\end{array}$ \\
1. Range of spectral types & G0 - M2 & B $5-\mathrm{F} 2$ \\
2. Am stars & 1 (o Leo) & $9(20 \%)$ \\
3. Range of periods & 14 days -65 years \\
& $<120$ days: $11(24 \%)$ \\
& $0.3-3$ years: $12(27 \%)$ \\
4. Triple systems & $>3$ years: $22(49 \%)$ \\
5. Eclipsing systems & 6, maybe $7(14 \%)$ \\
6. Astrometric orbits & $8(18 \%)$ \\
in addition to those in \# 5) & $8(18 \%)$ \\
** Except for $o$ Leo, the components are separated by the Hertzsprung gap. \\
\hline \hline
\end{tabular}

Our method is to obtain high $\mathrm{S} / \mathrm{N}$ spectra of a system at numerous orbital phases, particularly at or near nodes. The spectrum of the giant star is then subtracted away, pixel by pixel, from the spectrum of the binary by using the spectrum of a standard star of known spectral type. The best match indicates the spectral type and luminosity class of the giant in the binary. The residue is the spectrum of the hot component, which can then be classified, matched to synthetic spectra, and measured for RV. Knowing the RV of the giant on the respective dates from its SB1 orbit, we can in principle derive an SB2 orbit for the system. An example of how the procedure works is shown in Fig. 1. In this 


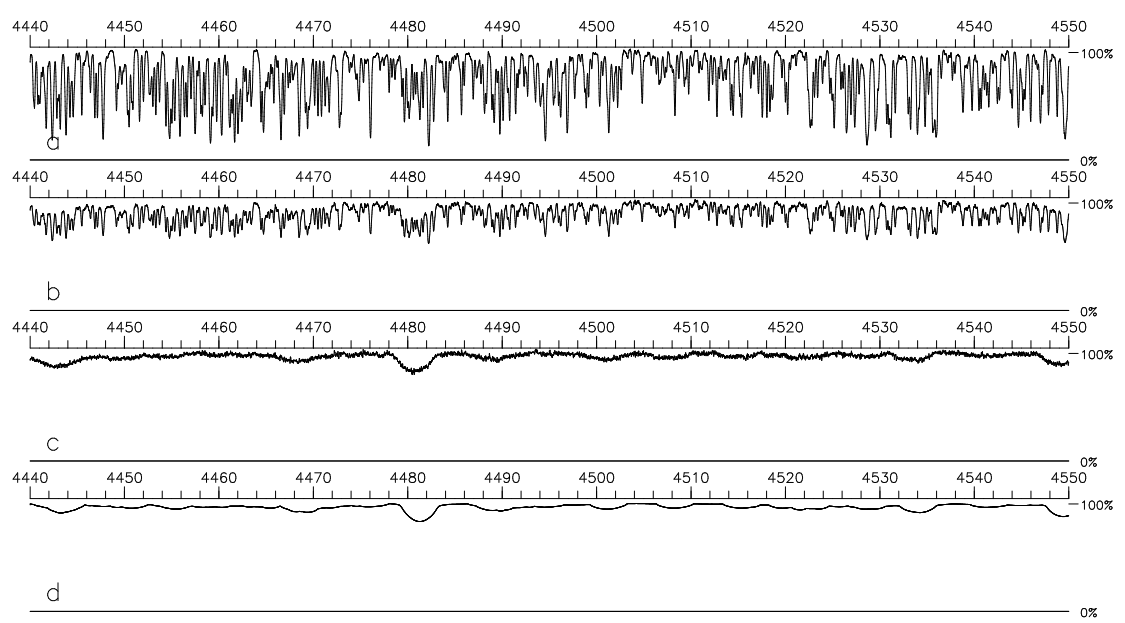

Figure 1. Separating component spectra of 45 Cnc by subtraction. The spectrum of an appropriate cool-giant standard, in panel (a), is subtracted point-by-point from the spectrum of the composite system in panel (b). The residue, seen in panel (c), is the spectrum of the hot dwarf secondary; it matches closely a synthetic spectrum, calculated in this case for $T_{\text {eff }} \doteq 8500, \log g=3.5$, solar abundances (but with $[\mathrm{Mg} / \mathrm{Fe}]$ raised by 0.3 dex), and blurred to mimic rotation of $130 \mathrm{~km} \mathrm{~s}^{-1}$.

figure, the spectrum of an appropriate G-giant spectrum, panel (a), is subtracted from the observed composite spectrum in panel (b), leaving the spectrum of the secondary star, 45 Cnc B - see panel (c) - as a residue. Panel (d) shows a synthetic spectrum, calculated with SPECTRUM ${ }^{1}$; it forms a close match to that of the hot dwarf in panel (c). The goodness of the match of the standard star is demonstrated by the general lack of 'mis-match' spikes (artefacts) in the residue. To optimize the procedure, it is important to align both the composite and the standard spectra in the same (stellar) rest-frame, rather than try to shift spectra to correct for stellar RVs.

\section{The good: unparallelled studies of stellar chromospheres}

One bonus of this research has been the discovery of the chromospheric eclipses which 9 of the sample manifest. All stars possess chromospheres. The solar chromosphere is relatively shallow compared to the diameter of the disk, but in a cool giant, especially a supergiant, it is markedly extensive. Near to eclipse, when the hot dwarf passes behind such a region, its radiation is absorbed selectively by the cool chromosphere, generating narrow features that contain highly valuable information about the conditions and chemistry of the giant's chromosphere -

${ }^{1}$ www.appstate.edu/grayro/spectrum/spectrum.html 


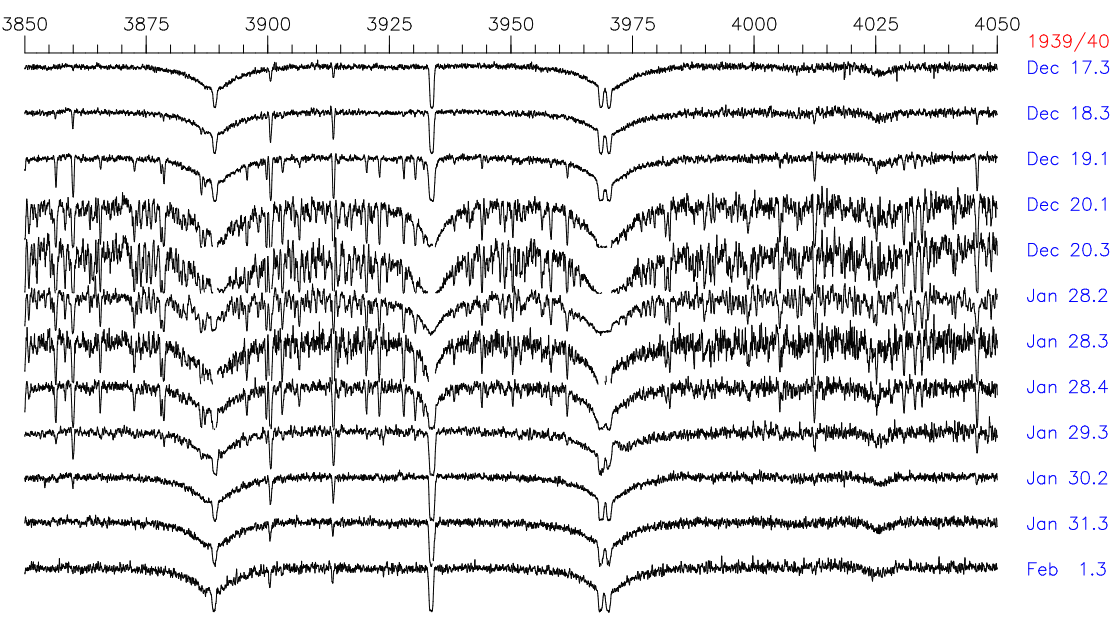

Figure 2. Sequence of selected chromospheric spectra, superimposed on spectra of the hot dwarf as it passes behind the chromosphere of the supergiant primary from ingress to egress on the dates shown. Total eclipse lasted for $\sim 36$ days; mid-eclipse was close to 1940 January 8. The changing strengths of the chromospheric lines as the dwarf aproaches, and then recedes from, the limb of the giant reflect the density gradient in the chromosphere.

information that is simply not attainable in any other way, so these eclipses are precious phenomena. $\zeta$ Aur, the first system in which the possibility of such eclipses was recognized, and detected by Guthnick (1934), has given its name to this sub-class. Analyses of the chromospheres of several $\zeta$ Aur systems indicate that chromospheres are by no means static or homogeneous, that they contain local concentrations of material and a lot of empty space, and that a series of eclipse spectra (see Fig. 2) differs at each eclipse from every other set. Those findings launched a fresh programme of investigation into the properties of the chromospheres of cool giants and supergiants. One fascinating outcome is the suspicion that cool giants undergo activity cycles analogous to the sunspot cycle; eclipses in 32 Cyg are grazing, and changes in the degree of chromospheric absorption at the pole of the supergiant primary in different eclipses are similar to the changing shape of the solar corona between sunspot maxima and minima.

\section{The bad: difficulties of analysis imposed by Nature}

The hot dwarf featured in Fig. 1 is rotating at $\sim 130 \mathrm{~km} \mathrm{~s}^{-1}$, fast enough to create a challenge for precise RV measurements. The subtraction procedure subtracts the signal but adds the noise, so even a well-exposed spectrum can exhibit considerable noise when (as often happens) over half of the signal has been subtracted. Taking multiple observations and co-adding them is a good 
solution in such binaries, but an early-A or a late-B dwarf does not have many lines in its spectrum anyway; the Ca II line is relatively blend-free and should in principle be a good for RG measurements, but it is often contamiated with an IS feature, making it unuseable for precise velocity measurements in practice.

\section{The really ugly}

The majority of the systems analyzed so far prove to have component stars whose properties - mass, luminosity and age - are in accordance with current stellar evolutionary theory (though it was a bit surprising to discover such a high percentage of triple systems). However, a few do not, and it is those misfits which should cause us to worry. The talk singled out two.

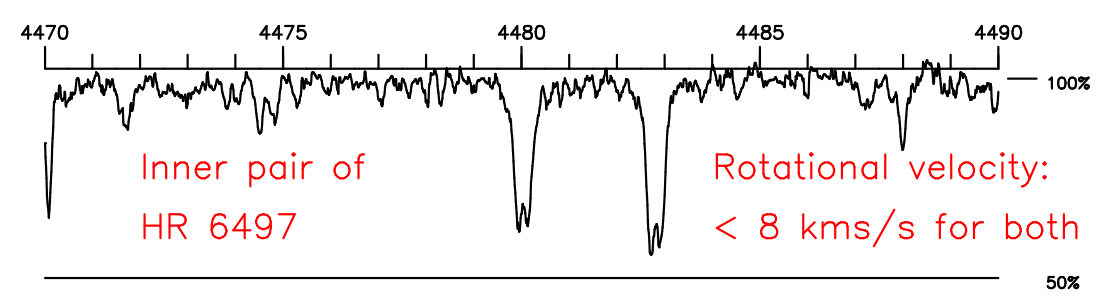

Figure 3. Portion of the secondary spectrum of HR 6497, showing the two component lines of Mg II near $\lambda 4481 \AA$. The lack of any visible rotational velocity in the line of sight cannot readily be explained without adopting some highly unlikely model.

(1) HR 6497 ( $P=3.3$ years) is an hierarchical triple system, whose inner pair form a tight binary $(P=3.75$ days); see Griffin \& Griffin (2012). What is strange is that the two secondary stars each have orbital velocities around 100 $\mathrm{km} \mathrm{s}^{-1}$ and yet they show no rotation at all. Fig. 3 reproduces a portion of the uncovered spectrum of the secondary system to show the strong, narrow $\mathrm{Mg}$ II lines at $\lambda 4481 \AA$ in each component, widely separated in velocity when near a node in the 3.75-day orbit. The Mg II line is actually a close doublet with a separation close to $0.2 \AA$, and its partial resolution as seen here is very puzzling as it indicates almost zero rotation of the stars along our line of sight. The inner orbit is tilted at about $72^{\circ}$, and it does not seem at all likely that the two stars should be tipped to that extent so that they appear face-on: the likelihood of such a coincidence must be vanishingly small, and additionally very unlikely to occur in a sample of only 45 systems.

(2) Another system, HD 193350, appears to be a simple binary; its SB2 orbait is illustrated in Fig. 4; see Griffin \& Griffin (2018). No trace of a third body has been detected, but the secondary proves to be more than twice as massive as the primary. There is no evidence of mass transfer in its history. The only feasible conclusion is a merger (e.g., of a pair that used to form a double secondary, 


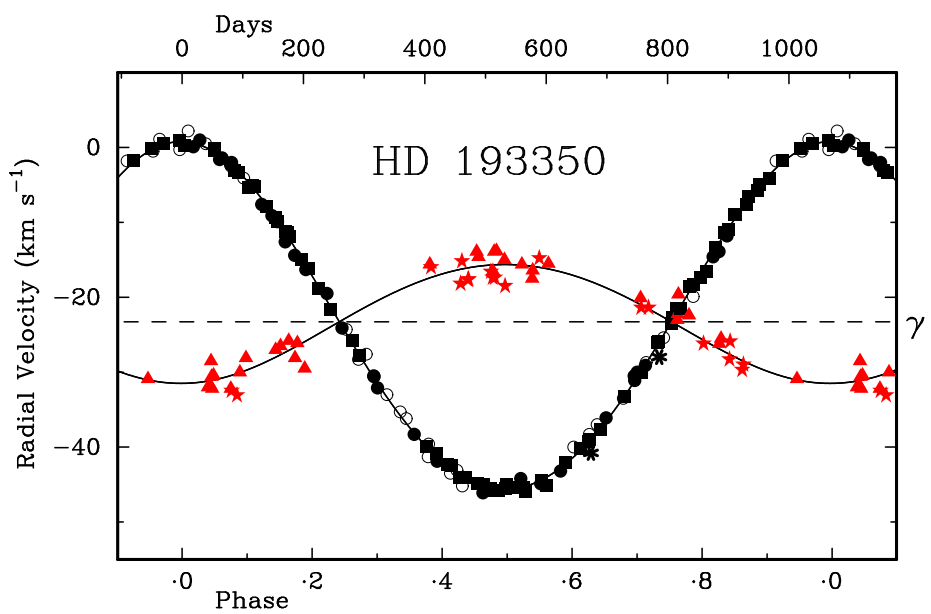

Figure 4. SB2 orbit for HD 193350. The orbit of the secondary (the less populated curve, featuring asterisks and filled triangles) indicates that the (unevolved) secondary star is substantially more massive than the (evolved) primary.

as in HR 6497 now). It must have occurred long enough ago for the resulting binary system to have become totally quiescent, because there is no evidence in the form of emission features that might be expected when such a violent event takes place.

(3) The secondary of HD 88021 is a confirmed Am star, but contrary to many assertions that Am stars undergo chemical separation by diffusion because they are held quiescent in tidally-locked binaries, HD $88021 \mathrm{~B}$ shows no sign that it is in a close binary - in fact, the only orbit involved combines it with its cool giant primary, and has $P=75$ years.

These problem cases need to worry us. If we cannot explain why they seem to defy some of the basic concepts of stellar astrophysics, why are we straining to understand all the other stars in the Galaxy?

Acknowledgements. I am very grateful both to the HAA (DAO) in Victoria and to the Local Organizing Committee for financial support that enabled me to take part in this conference.

\section{References}

Griffin, R. \& R.: 1986, JA\&A, 7, 4

Griffin, R.E.M. \& Griffin, R.F.: 2012, AN, 333, 613

Griffin, R.E.M. \& Griffin, R.F.: 2018, AN, 339, 586

Guthnick, P.: 1934, AN, 252, 13 